ESAIM: COCV 25 (2019) 54

https://doi.org/10.1051/cocv/2019060
ESAIM: Control, Optimisation and Calculus of Variations

www.esaim-cocv.org

\title{
ERRATUM TO: A VARIATIONAL APPROACH TO A STATIONARY FREE BOUNDARY PROBLEM MODELING MEMS
}

\author{
Philippe Laurençot ${ }^{1, *}$ And Christoph WAlkeR $^{2}$
}

\begin{abstract}
An incomplete argument in the proof of Theorem 3.4 from Ph. Laurençot and Ch. Walker [ESAIM: COCV 22 (2016) 417-438] is corrected.
\end{abstract}

Mathematics Subject Classification. 35J35, 35R35, 35Q74.

Received July 25, 2019. Accepted September 26, 2019.

We noticed a gap in the proof of Theorem 3.4 from [2] and the aim of this erratum is to provide a complete argument. Specifically, in Theorem 3.4 from [2], we derive the Euler-Lagrange equation satisfied by a minimizer $u$ of the functional

$$
\mathcal{E}_{m}(u):=\frac{\beta}{2}\left\|\partial_{x}^{2} u\right\|_{L_{2}(I)}^{2}+\frac{1}{2}\left(\tau+\frac{a}{2}\left\|\partial_{x} u\right\|_{L_{2}(I)}^{2}\right)\left\|\partial_{x} u\right\|_{L_{2}(I)}^{2}
$$

on the set

$$
\mathcal{A}_{\rho}:=\left\{u \in H_{D}^{2}(I): u \text { is even with }-1<u \leq 0 \text { and } \mathcal{E}_{e}(u)=\rho\right\},
$$

where $I:=(-1,1), \rho \in(2, \infty), H_{D}^{2}(I):=\left\{u \in H^{2}(I): u( \pm 1)=\partial_{x} u( \pm 1)=0\right\}$, and $\mathcal{E}_{e}$ is a non-negative nonlinear and nonlocal functional of $u$. The computation in [2] of the Euler-Lagrange equation, see equation (3.10) from [2], relies implicitly on the property that minimizers lie in the interior of $\mathcal{A}_{\rho}$, a property which is, however, not known a priori. Although knowing that minimizers are strictly greater than -1 , it is actually not known whether minimizers are negative (even though this property can be shown a posteriori, which was the main reason to include it in the definition of $\mathcal{A}_{\rho}$ ). This issue can be remedied by changing slightly the admissible set $\mathcal{A}_{\rho}$ on which the functional $\mathcal{E}_{m}$ is minimized. In fact, the non-positivity assumption in $\mathcal{A}_{\rho}$ is not needed and our analysis works equally well in the set

$$
\mathscr{A}_{\rho}:=\left\{u \in H_{D}^{2}(I): u \text { is even with }-1<u \text { and } \mathcal{E}_{e}(u)=\rho\right\} \text {. }
$$

Keywords and phrases: MEMS, stationary solution, constrained minimization.

${ }^{1}$ Institut de Mathématiques de Toulouse, UMR 5219, Université de Toulouse, CNRS, 31062 Toulouse Cedex 9, France.

${ }^{2}$ Leibniz Universität Hannover, Institut für Angewandte Mathematik, Welfengarten 1, 30167 Hannover, Germany.

* Corresponding author: laurenco@math.univ-toulouse.fr 
To be more precise, several results in [2] were derived for non-positive functions in

$$
\mathcal{K}^{s}:=\left\{u \in H_{D}^{s}(I):-1<u \leq 0 \text { on } I\right\}, \quad s \geq 1
$$

an assumption which is not required, as it suffices to work in

$$
S^{s}:=\left\{u \in H_{D}^{s}(I):-1<u \text { on } I\right\}, \quad s \geq 1 .
$$

For $u \in S^{1}$, one shall then rather define the function $b_{u}$ in equation (2.1) from [2] as

$$
b_{u}(x, z):=\left\{\begin{array}{cll}
\frac{1+z}{1+u(x)} & \text { for } & (x, z) \in \overline{\Omega(u)} \\
1 & \text { for } & (x, z) \in \overline{\Omega\left(M_{u}\right)} \backslash \overline{\Omega(u)}
\end{array}\right.
$$

where $\Omega\left(M_{u}\right):=I \times\left(-1, M_{u}+1\right)$ with $M_{u}:=\max \left\{0, \sup _{I} u\right\}$. Note that $b_{u}$ belongs to $H^{1}\left(\Omega\left(M_{u}\right)\right) \cap C\left(\overline{\Omega\left(M_{u}\right)}\right)$, which allows one to redefine $B_{u} \in H^{-1}\left(\Omega\left(M_{u}\right)\right.$ ) (i.e. the dual space of $H_{D}^{1}\left(\Omega\left(M_{u}\right)\right)$ ) in equation (2.2) from [2] by

$$
\left\langle B_{u}, \vartheta\right\rangle:=-\int_{\Omega\left(M_{u}\right)}\left[\varepsilon^{2} \partial_{x} b_{u} \partial_{x} \vartheta+\partial_{z} b_{u} \partial_{z} \vartheta\right] \mathrm{d}(x, z), \quad \vartheta \in H_{D}^{1}\left(\Omega\left(M_{u}\right)\right)
$$

Then Lemmas 2.1 and 2.2 from [2] remain true for $u \in S^{1}$ (instead of $u \in \mathcal{K}^{1}$ ) and Proposition 2.3 from [2] is actually valid for $u \in S^{2-\alpha}$ (instead of $u \in \mathcal{K}^{2-\alpha}$ ) when replacing equation (2.5) from [2] by

$$
\frac{1+z}{1+M_{u}} \leq \psi_{u}(x, z) \leq 1, \quad(x, z) \in \Omega(u) .
$$

Moreover, Propositions 2.6 and 2.7 from [2] are also true when replacing $\mathcal{K}^{1}$ by $S^{1}$. For later use, we note that Proposition 2.6 from [2] implies

$$
\mathcal{E}_{e}(u) \leq \mathcal{E}_{e}(0)=2 \quad \text { for } \quad u \in S^{1} \quad \text { with } \quad u \geq 0 \text { in } I .
$$

Also Lemma 2.8 from [2] remains true for $u \in S^{1}$ (instead of $u \in \mathcal{K}^{1}$ ), except that the lower bound on $\mathcal{E}_{e}(u)$ has to be replaced by

$$
\mathcal{E}_{e}(u) \geq \int_{-1}^{1} \frac{\mathrm{d} x}{1+u(x)} \geq \frac{2}{1+M_{u}}
$$

All other statements of Section 2 from [2] are not affected by these changes.

The minimization of $\mathcal{E}_{m}$ in Section 3 from [2] is now performed on the set $\mathscr{A}_{\rho}$ defined in (1) for a given $\rho \in(2, \infty)$. The statement of Proposition 3.1 from [2] remains true, as it is easily checked that its proof only relies on the continuity of the map $t \mapsto \mathcal{E}_{e}(t v)$ for $v \in \mathscr{A}_{\rho}$ established in Proposition 2.7 from [2], but not on its monotonicity (which is only true when $v$ is non-negative). Next, neither Proposition 3.2 from [2], nor Lemma 3.3 from [2] are affected by the change of $\mathcal{A}_{\rho}$ to $\mathscr{A}_{\rho}$. Therefore, in the proof of Theorem 3.4 from [2] we can use the same arguments to derive that, if $u \in \mathscr{A}_{\rho}$ is an arbitrary minimizer of $\mathcal{E}_{m}$ on $\mathscr{A}_{\rho}$, then $u \in H^{4}(D) \cap H_{D}^{2}(I)$, and there is a Lagrange multiplier $\lambda_{u} \in \mathbb{R}$ such that

$$
\beta \partial_{x}^{4} u-\left(\tau+a\left\|\partial_{x} u\right\|_{L_{2}(I)}^{2}\right) \partial_{x}^{2} u=-\lambda_{u} g(u), \quad x \in I,
$$


where $g(u):=\partial_{u} \mathcal{E}_{e}(u)$ is a non-negative functional of $u$, which belongs to $L_{2}(I)$. At this stage, since the nonpositivity of $u$ is not yet guaranteed, we need to employ a slightly different argument than in [2]. Indeed, we first assume for contradiction that $\lambda_{u} \leq 0$. Then $-\lambda_{u} g(u)$ is non-negative and it follows of (3) and Theorem 1.1 from [1] that $u>0$ in $I$. Hence $\rho=\mathcal{E}_{e}(u) \leq \mathcal{E}_{e}(0)=2$ by (2), contradicting $\rho \in(2, \infty)$. Consequently, $\lambda_{u}>0$ and $-\lambda_{u} g(u)$ is negative, so that we infer of (3) and Theorem 1.1 from [1] that $u<0$ in $I$. The remaining arguments in the proof of Theorem 3.4 from [2] are then the same.

Summarizing, the statement of Theorem 3.4 from [2] is correct, once $\mathcal{A}_{\rho}$ is replaced by $\mathscr{A}_{\rho}$. Thanks to the above analysis, Theorem 3.4 from [2] may be supplemented with the following result:

Corollary 1. Consider $\rho \in(2, \infty)$ and let $u \in \mathscr{A}_{\rho}$ be an arbitrary minimizer of $\mathcal{E}_{m}$ in $\mathscr{A}_{\rho}$. Then $u<0$ in $I$ and $u \in \mathcal{A}_{\rho}$. In addition,

$$
\mathcal{E}_{m}(u)=\min _{v \in \mathscr{A}_{\rho}} \mathcal{E}_{m}(v)=\min _{v \in \mathcal{A}_{\rho}} \mathcal{E}_{m}(v)
$$

\section{REFERENCES}

[1] Ph. Laurençot and Ch. Walker, Sign-preserving property for some fourth-order elliptic operators in one dimension or in radial symmetry. J. Anal. Math. 127 (2015) 69-89.

[2] Ph. Laurençot and Ch. Walker, A variational approach to a stationary free boundary problem modeling MEMS. ESAIM: COCV 22 (2016) 417-438. 